\title{
Biomimetic mineral coatings in dental and orthopaedic implantology
}

\author{
Yue-lian LIU (凶) ${ }^{1,2}$, Klaas de GROOT ${ }^{3}$, Ernst B. HUNZIKER ${ }^{2}$ \\ 1 Section of Oral Implantology, Department of Oral Function, Academic Center of Dentistry Amsterdam (ACTA), \\ Louwesweg 1, 1066 EA, Amsterdam, The Netherlands \\ 2 Center of Regenerative Medicine for Skeletal Tissues, University of Bern, Bern, Switzerland \\ 3 Yekimed AG, Bern, Switzerland
}

(C) Higher Education Press and Springer-Verlag 2009

\begin{abstract}
Biomimetic techniques are used to deposit coatings of calcium phosphate upon medical devices. The procedure is conducted under near-physiological, or "biomimetic", conditions of temperature and $\mathrm{pH}$ primarily to improve their biocompatibility and biodegradability of the materials. The inorganic layers generated by biomimetic methods resemble bone mineral, and can be degraded within a biological milieu.

The biomimetic coating technique involves the nucleation and growth of bone-like crystals upon a pretreated substrate by immersing this in a supersaturated solution of calcium phosphate under physiological conditions of temperature $\left(37^{\circ} \mathrm{C}\right)$ and $\mathrm{pH}(7.4)$. The method, originally developed by Kokubo in 1990, has since undergone improvement and refinement by several groups of investigators.

Biomimetic coatings are valuable in that they can serve as a vehicle for the slow and sustained release of osteogenic agents at the site of implantation. This attribute is rendered possible by the near-physiological conditions under which these coatings are prepared, which permits an incorporation of bioactive agents into the inorganic crystal latticework rather than their mere superficial adsorption onto preformed layers. In addition, the biomimetic coating technique can be applied to implants of an organic as well as of an inorganic nature and to those with irregular surface geometries, which is not possible using conventional methodologies.
\end{abstract}

Keywords biomimetic, biomineralization, osteoinduction, osteogenic agent, tissue engineering

Received December 5, 2008; accepted January 3, 2009

E-mail: y.liu@acta.nl

\section{Introduction}

The mineralization of biological tissues is a well-regulated process, which often occurs within a matrix of proteins and proteoglycans. The protein molecules may either completely envelop, or be themselves enveloped by, the mineral crystals. These organic molecules are essential not only for the initial deposition of mineral crystals, but also for their subsequent growth, orientation and organization. How, precisely, the proteins become sequestered by mineral crystals, determine their properties and participate in the crystallization process are questions that have been addressed, but not satisfactorily answered to date.

Anionic polypeptides and proteins that have been extracted from calcified tissues, such as nacre, are known to act as seeds for the growth of calcite or aragonite when they are attached to a substratum. Several groups of investigators have endeavoured to imitate this biological mineralization process by immersing polymeric substrates within metastable solutions of the inorganic components. And in one such attempt, calcium carbonate was successfully imprinted upon a surface of acidic acrylate. On the basis of nucleation studies involving Langmuir and selfassembled monolayers, crystal growth under the aforementioned in-vitro conditions has been attributed to a lattice match between the organic and the inorganic phases.

In orthopaedic and dental implantology, novel tools and techniques are being sought to improve the healing of bony tissue and osseointegration. Numerous attempts have been made to enhance the osteoconductivity of titanium prostheses. These include modifications in their surface properties and coating with a layer of calcium phosphate. Hydroxyapatite and related calcium phosphates are known to be biocompatible and osteoconductive $[1,2]$. Hydroxyapatite ceramics have been widely employed in 
orthopaedics for nearly 20 years. They are relatively cheap, non-toxic, minimally resorbed, of acceptable compressive strength, attach well to hard tissues and conduct bone apposition. The major drawback of hydroxyapatite ceramics is their low tensile strength [3-6]. Until recently, layers of calcium phosphate were deposited upon the surfaces of metal implants under physical conditions that were highly unphysiological. The methods employed have been various and include plasma spraying, high-velocity oxygen-fuel spraying, electrophoretic deposition, sol-gel deposition, hot isostatic pressing, frit enamelling, ionassisted deposition, pulsed-laser deposition, electrochemical deposition and sputter coating [1,6-16].

Hydroxyapatite is the most important representative of the bioactive calcium-phosphate ceramics. There is abundant evidence in the literature that sintered hydroxyapatite is well incorporated into living bone and that it does not undergo any significant biodegradation once it has become bonded to osseous tissue (a feature that may be disadvantageous as well as advantageous) $[5,6,17,18]$. Although the static mechanical strength of sintered hydroxyapatite is comparable to that of cortical bone, this material is prone to fatigue failure under conditions of high-tensile loading, which renders it unsuitable for applications in load-bearing situations. Titanium prostheses that have been coated with plasma-sprayed layers of hydroxyapatite have been used with promising results in joint reconstruction. The plasma-spraying technique, however, is conducted at extremely high temperatures $\left(>1000^{\circ} \mathrm{C}\right)$. The mineral layers generated are composed of large, partially molten hydroxyapatite particles, which are not only prone to delamination but are also poorly degraded in a biological milieu.

The technique whereby such mineral layers are produced has recently undergone a revolutionary change [19-21], which has had profound consequences for their potential to serve as drug-carrier systems. Hitherto, as aforementioned, calcium-phosphate layers were deposited upon the surfaces of metal implants under highly unphysiological physical conditions, which precluded the incorporation of bioactive agents. These agents could only be adsorbed, superficially, upon preformed layers. Such superficially adsorbed molecules are released too rapidly within a biological milieu to be effective in their osteoinductive capacity. Now, it is possible to deposit calcium-phosphate layers under near-physiological conditions of temperature and $\mathrm{pH}$ by the so-called biomimetic technique, during which process bioactive agents can be co-precipitated [22-24]. Since these molecules are integrated into the inorganic latticework, they are released gradually in vivo as the layer undergoes degradation. This feature enhances the capacity of these coatings to act as a carrier system for osteogenic agents, for example, bone morphogenetic proteins (BMPs) and the growth and differentiation factors (GDFs).

\section{Biomimetic mineral coatings}

Biomimetics, which literally means the mimicry of biology, is a branch of science in which biologists and engineers jointly endeavour to produce "bioinspired" materials that can be used for tissue engineering. This broad new field has ancient roots. The replacement of bodily parts dates back at least 2500 years to the time when the Etruscans substituted missing teeth with artificial ones carved from the bones of oxen. The first recorded use of dental amalgam to repair decayed teeth was in China in the year $659 \mathrm{AD}$. As human life expectancy continues to increase, the need for better coping with diseased, damaged or destroyed bodily components has heightened.

\subsection{Preparation technique}

Several years ago, attempts were made to coat medical devices with layers of calcium phosphate under more physiological or "biomimetic" conditions of temperature and $\mathrm{pH}[2,19,20,22,23,25]$, primarily to improve their biocompatibility and biodegradability. The inorganic layers generated by biomimetic methods are comparable to bone mineral, whereas those produced by conventional methods are composed of large, partially molten hydroxyapatite particles, which are not only prone to delamination but are also poorly degraded within a biological milieu.

The biomimetic coating technique involves the nucleation and growth of bone-like crystals upon a pretreated implant by immersing this within a supersaturated solution of calcium phosphate under physiological conditions of temperature $\left(37^{\circ} \mathrm{C}\right)$ and $\mathrm{pH}(7.4)$. The method, originally developed by Kokubo et al. in 1990 [19], has since been improved and refined by several groups of investigators [23,26-32].

The implant is first immersed within five-timesconcentrated simulated body fluid under high-nucleation conditions, viz., in the presence of $\mathrm{Mg}^{2+}[(7.5 \mathrm{~mol} / \mathrm{L})$ see Table 1], to inhibit crystal growth, for $24 \mathrm{~h}$ at $37^{\circ} \mathrm{C}$. A thin $(<3-\mu m$-thick), dense and amorphous layer of calcium phosphate is thereby uniformly deposited upon the implant surface, and this serves as a seeding substratum for the subsequent growth of a substantial (30- to 50- $\mu \mathrm{m}$ thick) crystalline latticework. The latter is prepared by immersing the coated implant within a supersaturated

Table 1 Millimolar compositions of human blood plasma (HBP), simulated body fluid $(\mathrm{SBF})$, five-times-concentrated $\mathrm{SBF}(\mathrm{SBF} \times 5)$ and supersaturated calcium-phosphate solution (CPS)

\begin{tabular}{lccrccc}
\hline & $\mathrm{Na}^{+}$ & $\mathrm{Mg}^{2+}$ & \multicolumn{1}{c}{$\mathrm{Ca}^{2+}$} & \multicolumn{1}{c}{$\mathrm{Cl}^{-}$} & $\mathrm{HPO}_{4}^{2-}$ & $\mathrm{HCO}_{3}^{-}$ \\
\hline HBP & 142.0 & 1.5 & 2.5 & 103.0 & 1.0 & 27.0 \\
SBF & 146.7 & 1.5 & 2.5 & 147.8 & 1.0 & 4.2 \\
$\mathrm{SBF} \times 5$ & 733.5 & 7.5 & 12.5 & 740.0 & 5.0 & 21.0 \\
CPS & 140.4 & 0 & 3.1 & 142.9 & 1.86 & 0 \\
\hline
\end{tabular}



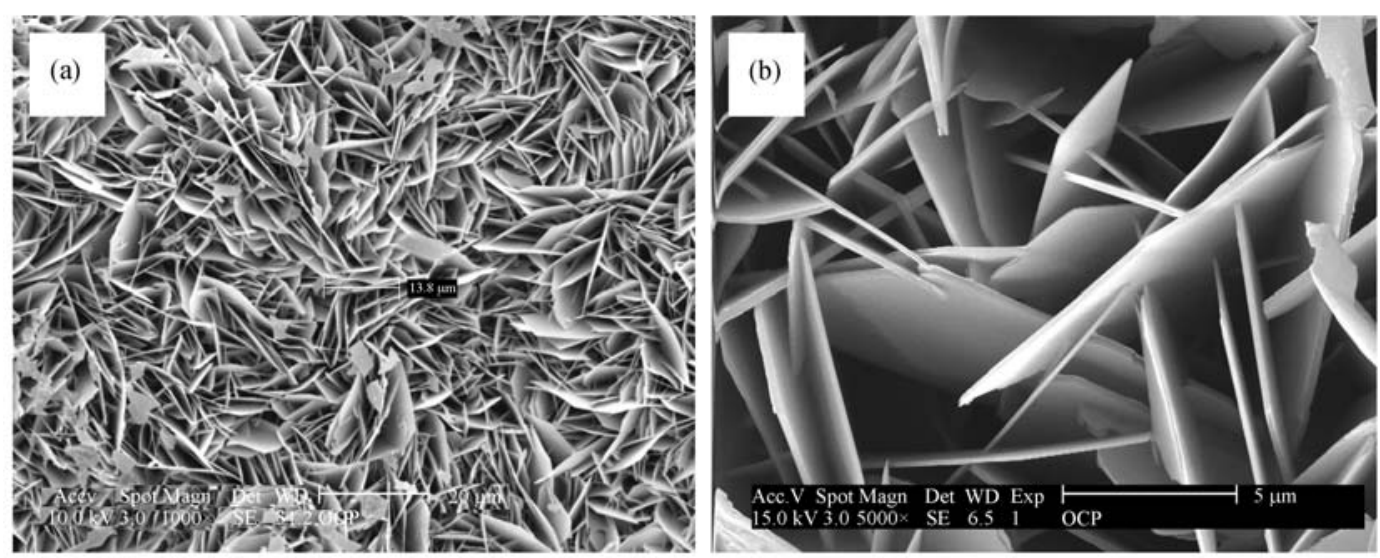

Fig. 1 Scanning electron micrographs of an octacalcium-phosphate coating at (a) low and (b) high magnifications

solution of calcium phosphate [(pH 7.4) Table 1] for $48 \mathrm{~h}$ at $37^{\circ} \mathrm{C}$. Under these conditions, the crystalline latticework is composed of octacalcium phosphate (Fig. 1).

The crystal structure of the inorganic latticework can be modified by changing the ionic composition of the coating solution, by increasing its temperature, or by prolonging the duration of the coating period.

The biomimetic coating process is simple to perform and cost-effective, and can be applied even to heat-sensitive, non-conductive porous materials of large dimensions with complex surface geometries. An additional advantage of the biomimetic method is that biologically active molecules, such as osteogenic agents, can be coprecipitated with the inorganic components. As a consequence, the proteins are truly incorporated into the crystal latticeworks and not merely deposited upon their surfaces. In forming an integral part of the calcium-phosphate coatings, the protein molecules are liberated not in a single burst (as when superficially adsorbed), but gradually, which bodes well for an enduring osteogenic effect at the implantation site. The disadvantage of such coatings is that they are of low cohesiveness and are degradable, both in vitro and in vivo, and adhere poorly to the underlying substrate. However, in a clinical situation, their biodegradability could be advantageous, particularly if they are serving as a vehicle for drug delivery.

\subsection{Characterization of mineral coatings}

\subsubsection{Coating thickness}

The thickness of coatings (up to $100 \mu \mathrm{m}$ ) can be measured in situ using a magnetic induction probe (Electrophysik minitest 2100, Germany).

\subsubsection{Fourier-transform infrared spectroscopy}

In preparation for Fourier-transform infrared spectroscopy, the coatings are peeled away from the carriers and then mixed with potassium bromide $(0.6-0.8 \mathrm{mg}$ of sample material per $280 \mathrm{mg}$ of potassium bromide). By means of this technique, the types of chemical bonds existing within the coatings can be established.

\subsubsection{Scanning electron microscopy and energy-dispersive} $\mathrm{X}$-ray spectroscopy

Prior to examination in a scanning electron microscope, the coated carriers are sputtered with carbon particles to a thickness of 12-16 nm. Scanning electron microscopy yields information on the geometry of the crystals of which the coatings are composed, whereas energy-dispersive Xray spectroscopy furnishes data on their chemical structure.

\subsubsection{Typical results achieved using the standard} biomimetic coating process

Mineral layers are typically $10-50 \mu \mathrm{m}$ in thickness. Scanning electron micrographs reveal coatings of octacalcium phosphate to be composed of thin, plate-like crystals (Fig. 1); those of carbonated apatite have an amorphous or a nano-crystalline appearance (Fig. 2).

Fourier-transform infrared spectroscopy discloses coatings of carbonated apatite to manifest two prominent absorption bands at 1410 and $1460 \mathrm{~cm}^{-1}$. Two $\mathrm{C}-\mathrm{O}$ bands stemming from $\mathrm{CO}_{3}^{2-}$ groups appear at 1470 and $1425 \mathrm{~cm}^{-1}$, $\mathrm{P}-\mathrm{O}$ bands from $\mathrm{PO}_{4}^{3-}$ groups at $1115,1042,970,610$ and $570 \mathrm{~cm}^{-1}$, and $\mathrm{P}-\mathrm{OH}$ bands from $\mathrm{HPO}_{4}^{2-}$ groups at 920 and $870 \mathrm{~cm}^{-1}$. Coatings of octacalcium phosphate are characterized by bands at 906 and $852 \mathrm{~cm}^{-1}$ [29].

\subsection{Biological functionalization of mineral coatings}

Although biomimetic calcium-phosphate coatings improve the osteoconductivity of metal implants, they do not render them osteoinductive, a feature that is required to expedite the osteogenic process and to accelerate implant 

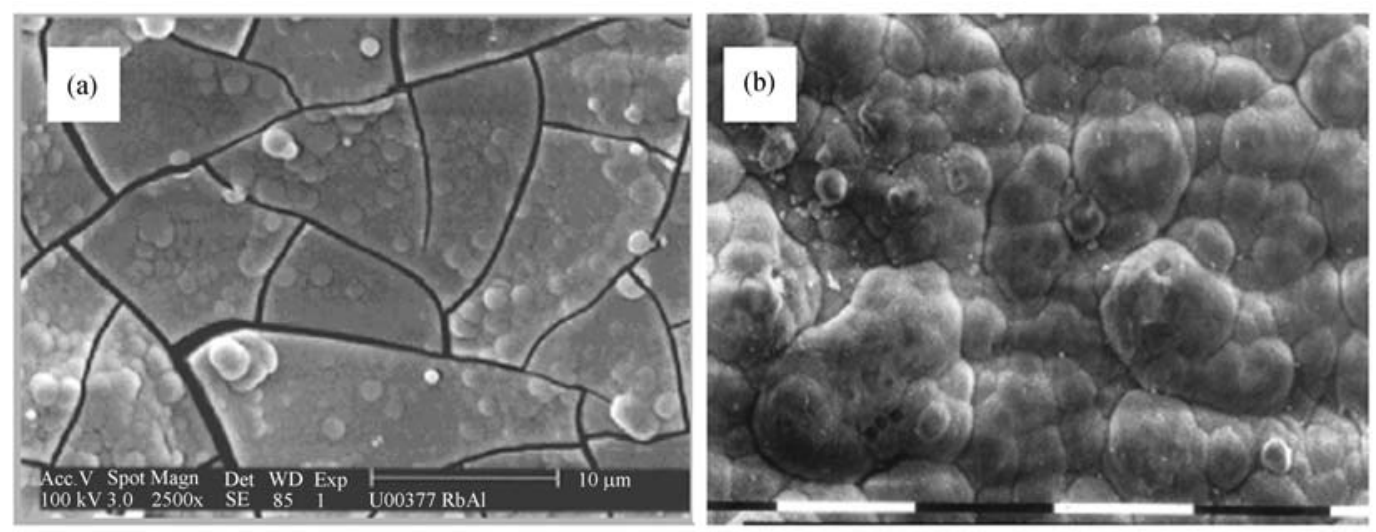

Fig. 2 Scanning electron micrographs of (a) an amorphous and (b) a nano-crystalline layer of carbonated apatite

integration. This property can be conferred by the introduction of an osteogenic agent [33-37]. Using conventional coating methods, osteogenic agents can be deposited only superficially upon preformed coatings, either by adsorption [33,38-40], by binding to biofunctional proteins [41], or by chemical treatment [42]. The disadvantage of this mode of attachment is that the biologically active molecules are released rapidly upon exposure to a physiological environment. Consequently, their osteogenic effects are short-lived. Using the biomimetic technique, osteogenic agents can be incorporated into the crystal latticework of the coatings. Consequently, they are released more slowly over a longer time-span.

\section{Biomimetic mineral coatings as a delivery vehicle for osteogenic agents}

The advent of the biomimetic coating technique has broadened the potential of calcium-phosphate layers to serve as a carrier system for osteogenic agents, thereby rendering them osteoinductive as well as osteoconductive. Granted this facility, investigators must select an appropriate osteogenic agent.

Members of the transforming growth factor superfamily (TGF- $\beta$ s), such as GDFs and especially BMPs, probably represent the most promising candidates for this purpose. Interest in this latter group of agents dates back to 1964. In this year, Urist [43] demonstrated that demineralized, dried and pulverized bone could stimulate the formation of osseous tissue at an ectopic site (muscle) in rabbits. The osteogenic fraction of the bone matrix was later isolated and subjected to amino-acid sequencing. This analysis revealed BMPs to be structurally similar to TGF- $\beta$ s, and they are now classed as a subgroup of this superfamily [44-47]. More than 15 of the 30 odd members have been isolated and synthesized by recombinant DNA technology [48-50].

Interest in BMPs obviously preceded the advent of the biomimetic coating technology, and various materials have thus already served as their carriers. These include collagen, synthetic and natural ceramics, demineralized bone matrix and polyglycolic acid [47,51-56]. Human recombinant BMP-2 used in conjunction with each of these carrier systems is released in two kinetically distinct phases: an initial rapid one of a few hours' duration and a second slower one spanning several weeks. Collagen retains the largest fraction of BMP-2 during the initial phase and synthetic hydroxyapatite particles the smallest $(10 \%)$. The other carriers retain between $30 \%$ and $50 \%$ of their load during this phase. With the exception of synthetic hydroxyapatite, the mineral-based carriers retain the largest fraction of BMP-2 during the second phase, thereby reflecting their high affinity for this agent. However, none of the materials serve as an optimal drugdelivery system.

The potential of biomimetic calcium-phosphate coatings to serve as a carrier system for BMP-2 is now being investigated by our group [57-59]. The drug has been successfully co-precipitated with the inorganic components and, thus incorporated, retains its biological activity in vitro $[58,59]$. Experiments using an ectopic (subcutaneous) model for bone formation in rats have revealed BMP-2 thus borne to be osteogenically potent. Furthermore, bone-forming activities are sustained for a considerable period of time, which indicates that BMP-2 is indeed released gradually (as anticipated) and not in a single rapid burst [57].

Instead of incorporating BMP-2 itself into a carrier system, the drug could be substituted by a plasmid containing the gene for the agent. The rationale behind such an undertaking would be that whilst a protein is ultimately degraded, a gene would become incorporated into a host cell and remain active for a longer period [60$62]$. The gene for BMP-2 has been delivered to orthotopic sites in rats and dogs via viral and non-viral carriers $[48,63]$; it has not yet been incorporated into biomimetic implant coatings. 


\section{In-vitro and in-vivo testing of the osteogenic potential of BMP-2-functionalized biomimetic coatings}

\subsection{In-vitro testing}

Titanium-alloy implants were coated biomimetically with a layer of calcium phosphate into which different amounts of BMP-2 were incorporated (1-3 $\mu \mathrm{g} / \mathrm{implant})$. The osteogenic potency of BMP-2 thus incorporated was assessed by monitoring the alkaline-phosphate activity of rat bone-marrow stromal cells that were cultured directly upon the coated implants. BMP-2 that was incorporated into the calcium-phosphate coatings was more potent in stimulating the alkaline-phosphatase activity of bonemarrow stromal cells that were cultured thereupon than was the freely suspended drug in stimulating the alkalinephosphatase activity of the same cell population that was cultured on a plastic surface. Hence, the osteogenic potency of BMP-2 is not only retained but enhanced by its biomimetic incorporation into a calcium-phosphate layer, probably by virtue of a localized concentration effect.

\subsection{Ectopic model in rats}

We investigated the potential of BMP-2-functionalized calcium-phosphate coatings to induce and sustain bone formation at an ectopic (subcutaneous) site in rats over a period of 5 weeks. For this purpose, one experimental and three control groups were established: titanium-alloy implants bearing a co-precipitated layer of calcium phosphate and BMP-2 (experimental group); naked titanium-alloy discs (negative control for the effects of a calcium-phosphate layer and of BMP-2); titanium-alloy discs bearing a layer of calcium phosphate (negative control for the effects of BMP-2); and titanium-alloy discs bearing a layer of calcium phosphate and superficially adsorbed BMP-2 (positive control for superficially adsorbed BMP-2). Six implants per group were implanted subcutaneously in the dorsal region of rats, two per animal. Samples were retrieved at 7-day intervals over a period of 5 weeks for histological and histomorphometric analyses. Ectopic bone formation occurred only in the experimental group of animals (Fig. 3). Bone tissue first became apparent 2 weeks after implantation and thereafter was deposited continually until the end of the 5-week monitoring period. Ossification occurred intramembranously (directly), there being virtually no evidence of an endochondral mechanism having been followed. The total volume of bone formed increased significantly $(p<0.005)$ from $5.8 \mathrm{~mm}^{3}$ per implant after the second week to 10.2 $\mathrm{mm}^{3}$ per implant after the third, when the net daily rate of bone formation was the highest $\left(0.8 \mathrm{~mm}^{3}\right.$ per implant per day). Bone tissue was deposited not only upon the implant surfaces but also at some distance therefrom, maximally at
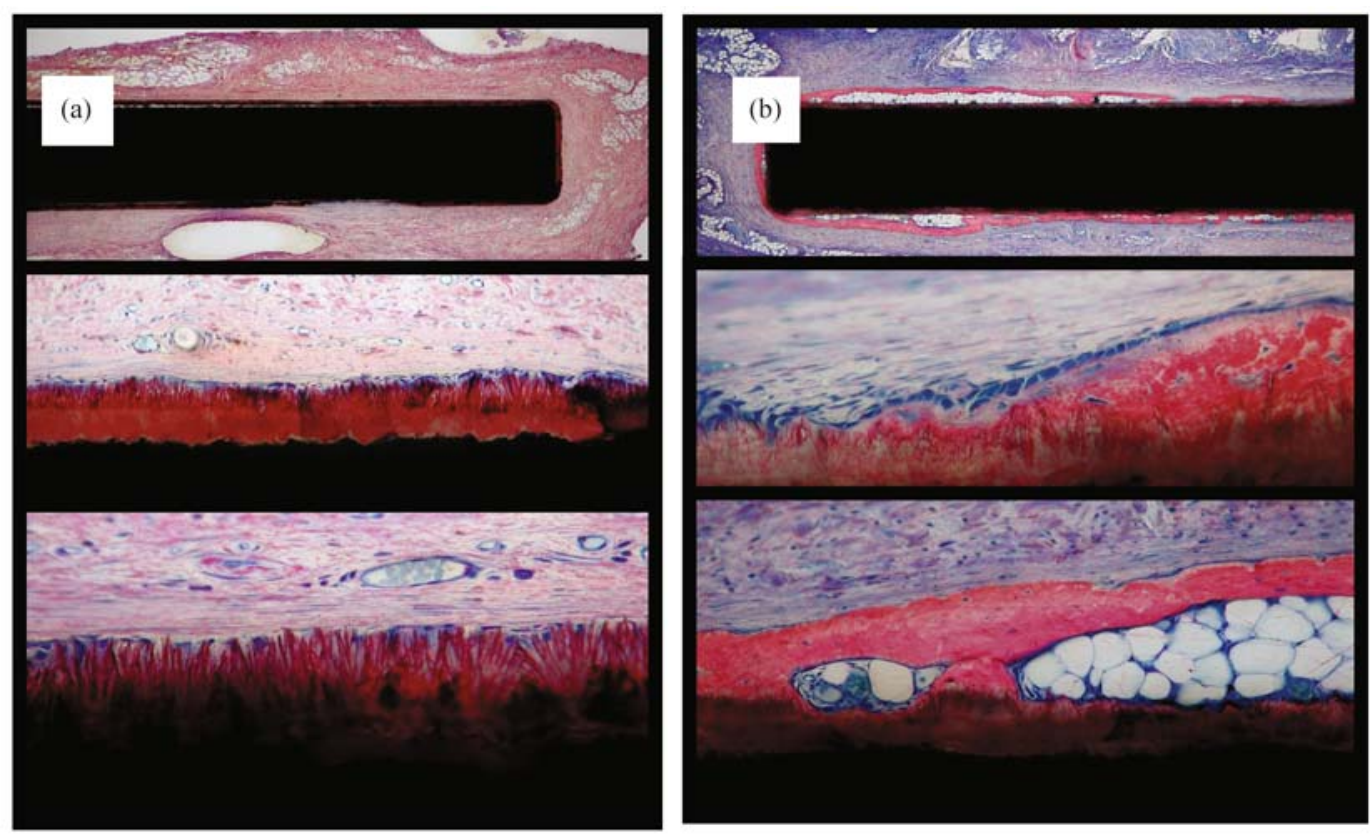

Fig. 3 (a) Light micrographs of a vertically-sectioned titanium-alloy disc bearing a calcium-phosphate coating, 5 weeks after implantation at a subcutaneous site in a rat (low, intermediate and high magnifications). No osseous tissue has been deposited. (b) Light micrographs of a vertically-sectioned titanium-alloy disc bearing a BMP-2-functionalized calcium-phosphate coating, 5 weeks after implantation at a subcutaneous site in a rat (low, intermediate and high magnifications). The upper image reveals the surface of the implant to be covered with osseous tissue. In the middle image, osteoblasts are seen to align the bone surface in an epithelium-like manner. The lower panel reveals bone-marrow tissue to contact the coating directly. 
$340 \mu \mathrm{m}$ by the end of the third week. This distance had decreased to $200 \mu \mathrm{m}$ by the end of the fifth week, indicating that bone remodelling processes were underway, which would be a normal occurrence under physiological conditions. The biomimetic coating was degraded gradually during the course of the 5-week monitoring period, by the end of which time, one third of its volume remained. The implication is, that one third of the initially incorporated amount of BMP-2 (1.7 $\mu \mathrm{g}$ per implant) likewise remained, and that bone formation could have continued for several more weeks after the end of the experimental period. The findings of this study demonstrate that when BMP-2 is incorporated biomimetically into calcium-phosphate coatings at a low pharmacological dose, it is capable not only of inducing the formation of bone at an ectopic site but also of sustaining osteogenic activity for a considerable period of time.

\subsection{Orthotopic model in miniature pigs}

To clarify the potential beneficial and adverse effects of BMP-2 on the osteoconductivity of titanium-alloy implants, we considered two basic types of surface: a sandblasted, acid-etched one and a sand-blasted, acid-etched one coated with a layer of calcium phosphate. These two surfaces served as the base-line controls. In the first category, BMP-2 was adsorbed directly onto the metal surface; in the second, it was either adsorbed onto, incorporated into, or incorporated into and adsorbed onto the calcium-phosphate coating. The implants were inserted into the partially edentulous maxillae of adult Goettingen miniature pigs. The neoformation of bone both along the implant surface and up to a distance of $175 \mu \mathrm{m}$ therefrom (the immediate peri-implant space) was monitored quantitatively 1, 2, and 3 weeks after surgery, viz., during the early phase of healing when any interference with the osteoconductivity of the implants would be apparent.

Our findings revealed the osteoconductivity of the implants to be greatly influenced by BMP-2 and its mode of delivery. The net volume of bone deposited within the immediate vicinity of the coated implants (up to $175 \mu \mathrm{m}$ therefrom) could be increased by the presence of BMP-2 during the first two postoperative weeks, but was decreased by its presence during the third. Bone coverage of the coated implants was lowered by the presence of BMP-2 at each weekly juncture. The net volume of bone deposited both directly upon and within the immediate vicinity of uncoated implants was lowered by the presence of BMP-2 at each time-point. In general, implant osteoconductivity was most severely compromised when BMP-2 was present as an adsorbed depot (subject to burstrelease kinetics), and less so when it was delivered via a slow-release system (incorporated into a calciumphosphate coating).

Data relating to the degradation of implant coatings are summarized in Table 2. Coatings bearing incorporated and adsorbed BMP-2 underwent the most extensive degradation during the 3-week monitoring period, followed by those bearing only an incorporated depot of the drug. Coatings bearing no BMP-2 underwent the least degradation during the 3 -week monitoring period, followed by those bearing only an adsorbed depot of the drug.

Table 2 Changes in coating thickness during the 3-week monitoring period [Mean values $(n=6)$ are represented together with the standard error of the mean]

\begin{tabular}{ccccc}
\hline Mode of BMP-2 loading & \multicolumn{4}{c}{ Coating thickness $/ \mu \mathrm{m}$} \\
& & {$[$ initial coating thickness $=(54.7 \pm 8.73) \mu \mathrm{m}]$} \\
\hline Incorporated & Adsorbed & Week 1 & Week 2 & Week 3 \\
\hline+ & - & $22.26 \pm 4.26$ & $2.58 \pm 1.36$ & $1.08 \pm 0.45$ \\
- & + & $45.53 \pm 4.36$ & $16.82 \pm 5.45$ & $13.35 \pm 4.17$ \\
+ & + & $14.63 \pm 2.46$ & $6.52 \pm 2.82$ & $0.46 \pm 0.2$ \\
- & - & $52.00 \pm 1.83$ & $27.72 \pm 7.69$ & $15.31 \pm 3.14$ \\
\hline
\end{tabular}

\section{Biological functionalization of biomimetic mineral coatings}

That calcium-phosphate layers are biocompatible and osteoconductive is well known. Indeed, it was for these attributes that this type of coating was chosen to act as a carrier for BMP-2. Although BMP-2 is itself osteoinductive, its osteogenic potency is markedly enhanced after incorporation into biomimetic calcium-phosphate coatings [58]. This finding is accounted for by the drug's slow rate of release from these coatings over a protracted period of time. BMP-2 is liberated gradually because it forms an integral part of the inorganic latticework. BMP-2 that has been deposited superficially upon the surfaces of preformed calcium-phosphate coatings is released in a single rapid burst. Since the drug is highly water-soluble, it diffuses away from the implantation site too quickly to exert an osteogenic effect [57]. The forces governing protein adsorption onto the surface of a preformed calcium-phosphate coating are believed to be chiefly electrostatic in nature [64], but the mechanisms underlying the incorporation of protein molecules during their coprecipitation with calcium phosphate are more complex and poorly understood. Protein molecules possibly participate in the formation of the three-dimensional crystal latticework, or at least influence its growth. They may act as tied molecules, in which case they would be effectively bonded, or serve as seeds for crystallization and possibly influence the number of nucleation sites generated $[65,66]$. Protein molecules may also be aggregated and penetrated by mineral ions during the formation of a coating. Although bovine serum albumin influences the structure of the calcium-phosphate latticework into which it is incorporated, BMP-2 does not have this effect. Hence, the mechanism of incorporation may be different in each case. However, the presence of incorporated BMP-2 does 
influence the physical properties of biomimetic coatings. In vivo, proteins are known to strengthen mineralized tissue and to stabilize its mineral contents. And in our studies, the rate of release of calcium ions from biomimetic coatings was indeed slowed down by the presence of incorporated BMP-2, which may improve the coating's attachment to the underlying titanium-alloy implant. Indeed, coatings that are functionalized with BMP-2 have been shown to be mechanically more cohesive than unfunctionalized ones. BMP-2 that has been incorporated into the body of the inorganic latticework possibly reinforces the coating by surrounding the brittle calciumphosphate crystals, in analogy to the situation occurring in dental enamel. This property of mechanical cohesiveness is important during the surgical implantation procedure, which generates high shear forces that could rupture the coatings. That the coated implants furnished a mechanically stable environment in vivo was evidenced by the finding that ectopic bone formation in rats occurred by intramembranous growth. In previous studies that have made use of this model, ossification has followed a predominantly endchondral course, which requires a less stable mechanical environment. Our own studies with the model [67] also yielded evidence of the high biocompatibility of the BMP-2-functionalized coatings, in that bonemarrow tissue was sometimes observed to contact these directly, without intervening bone (see Fig. 3). Bonemarrow tissue contains immunocompetent cells, which are very sensitive to foreign material. Dental implants bearing BMP-2-functionalized calcium-phosphate coatings were more rapidly osseointegrated at an orthotopic site in adult miniature pigs than were uncoated ones [68].

\section{Conclusions}

BMP-2 can be incorporated in a dose-dependent manner into biomimetic calcium-phosphate coatings that are deposited upon titanium-alloy implants. The incorporated BMP-2 is released gradually (over a period of several weeks) into the surrounding milieu wherein it retains its biological activity. At an ectopic site in rats, BMP-2 is released from the coatings and induces the formation of bone not only upon the implant surface but also within its immediate surroundings. At an orthotopic dental site in adult miniature pigs, coatings bearing an incorporated depot of BMP-2 enhance implant osseointegration.

Biomimetic mineral coatings are valuable in that they can serve as a vehicle for the slow, sustained release of an osteogenic agent at an implantation site. This facility relates to the near-physiological conditions under which the mineral coatings are prepared, which permits the incorporation of biological agents into the inorganic crystal latticework rather than their mere superficial adsorption upon preformed layers. Furthermore, the biomimetic coating technique can be applied to implants of an organic as well as of an inorganic nature and to those with irregular surface geometries, which is not possible using methodologies.

Acknowledgements This work was supported by the Swiss National Science Foundation and the AO/ASIF-Foundation, Switzerland.

\section{References}

1. de Groot K. Calciumhydroxylapatite. Journal of Oral Implantology, 1986, 12(3): 485-489

2. de Groot K. Hydroxylapatite coated implants. Journal of Biomedical Materials Research, 1989, 23(11): 1367-1371

3. Kitsugi T, Yamamuro T, Nakamura T, et al. Four calcium phosphate ceramics as bone substitutes for non-weight-bearing. Biomaterials, 1993, 14(3): 216-224

4. Klein C P, Driessen A A, de Groot K. Relationship between the degradation behavior of calcium phosphate ceramics and their physical-chemical characteristics and ultrastructural geometry. Biomaterials, 1984, 5(3): 157-160

5. Klein C P, Wolke J G C, Deblieckhogervorst J M A, et al. Features of calcium phosphate plasma-sprayed coatings: an in vitro study. Journal of Biomedical Materials Research, 1994, 28(8): 961-967

6. Klein C P, Patka P, Wolke J G C, et al. Long-term in vivo study of plasma-sprayed coatings on titanium alloys of tetracalcium phosphate, hydroxyapatite and alpha-tricalcium phosphate. Biomaterials, 1994, 15(2): 146-150

7. de Groot K, Geesink R, Klein C P, et al. Plasma sprayed coatings of hydroxylapatite. Journal of Biomedical Materials Research, 1987, 21(12): 1375-1381

8. Wolke J G C, Vandijk K, Schaeken H G, et al. Study of the surface characteristics of magnetron-sputter calcium phosphate coatings. Journal of Biomedical Materials Research, 1994, 28(12): 14771484

9. Wolke J G C, van der Waerden J P C M, de Groot K, et al. Stability of radiofrequency magnetron sputtered calcium phosphate coatings under cyclically loaded conditions. Biomaterials, 1997, 18(6): 483488

10. Wolke J G C, de Groot $\mathrm{K}$, Jansen J A. In vivo dissolution behavior of various RF magnetron sputtered Ca-P coatings. Journal of Biomedical Materials Research, 1998, 39(4): 524-530

11. Wolke J G C, de Groot K, Jansen J A. Subperiosteal implantation of various RF magnetron sputtered Ca-P coatings in goats. Journal of Biomedical Materials Research, 1998, 43(3): 270-276

12. Jansen J A, Wolke J G, Swann S, et al. Application of magnetron sputtering for producing ceramic coatings on implant materials. Clinical Oral Implants Research, 1993, 4(1): 28-34

13. Vehof J W M, van den Dolder J, de Ruijter J E, et al. Bone formation in CaP-coated and noncoated titanium fiber mesh. Journal of Biomedical Materials Research, 2003, 64A(3): 417-426

14. Agata De Sena L, Calixto De Andrade M, Malta Rossi A, et al. Hydroxyapatite deposition by electrophoresis on titanium sheets with different surface finishing. Journal of Biomedical Materials Research, 2002, 60(1): 1-7

15. Wang J, de Boer J, de Groot K. Preparation and characterization of 
electrodeposited calcium phosphate/chitosan coating on $\mathrm{Ti}_{6} \mathrm{Al}_{4} \mathrm{~V}$ plates. Journal of Dental Research, 2004, 83(4): 296-301

16. Wang J, Layrolle P, Stigter M, et al. Biomimetic and electrolytic calcium phosphate coatings on titanium alloy: physicochemical characteristics and cell attachment. Biomaterials, 2004, 25(4): 583592

17. Zhang H Q, Li S P, Yan Y H, et al. Dissolution behavior of hydroxyapatite coating by hydrothermal method: an in vitro study. Biomedical Materials and Engineering, 2000, 10(3-4): 213-219

18. Schliephake H, Scharnweber D, Dard M, et al. Biological performance of biomimetic calcium phosphate coating of titanium implants in the dog mandible. Journal of Biomedical Materials Research, 2003, 64A(2): 225-234

19. Kokubo T, Kushitani H, Sakka S, et al. Solutions able to reproduce in vivo surface-structure changes in bioactive glass-ceramic A-W. Journal of Biomedical Materials Research, 1990, 24(6): 721-734

20. Kokubo T. Bioactive glass ceramics: properties and applications. Biomaterials, 1991, 12(2): 155-163

21. Li P. Bioactive ceramic coating and method. US Patent, 6139583, 2000

22. Wen H B, de Wijn J R, van Blitterswijk C A, et al. Incorporation of bovine serum albumin in calcium phosphate coating on titanium. Journal of Biomedical Materials Research, 1999, 46(2): 245-252

23. Liu Y L, Layrolle P, de Bruijn J, et al. Biomimetic coprecipitation of calcium phosphate and bovine serum albumin on titanium alloy. Journal of Biomedical Materials Research, 2001, 57(3): 327-335

24. Liu Y, Hunziker E B, Layrolle P, et al. Remineralization of demineralized albumin-calcium phosphate coatings. Journal of Biomedical Materials Research Part A, 2003, 67A(4): 1155-1162

25. de Groot K, Wolke J G, Jansen J A. Calcium phosphate coatings for medical implants. Proceedings of the Institution of Mechanical Engineers Part H, 1998, 212(2): 137-147

26. Barrere F, van Blitterswijk C A, de Groot $\mathrm{K}$, et al. Influence of ionic strength and carbonate on the Ca-P coating formation from $\mathrm{SBF} \times 5$ solution. Biomaterials, 2002, 23(9): 1921-1930

27. Barrere F, van Blitterswijk C A, de Groot $\mathrm{K}$, et al. Nucleation of biomimetic Ca-P coatings on $\mathrm{Ti}_{6} \mathrm{Al}_{4} \mathrm{~V}$ from a $\mathrm{SBF} \times 5$ solution: influence of magnesium. Biomaterials, 2002, 23(10): 2211-2220

28. Barrere F, Layrolle P, van Blitterswijk C A, et al. Biomimetic coatings on titanium: a crystal growth study of octacalcium phosphate. Journal of Materials Science - Materials in Medicine, 2001, 12(6): 529-534

29. Barrere F, Layrolle P, van Blitterswijk C A, et al. Biomimetic calcium phosphate coatings on $\mathrm{Ti}_{6} \mathrm{Al}_{4} \mathrm{~V}$ : a crystal growth study of octacalcium phosphate and inhibition by $\mathrm{Mg}^{2+}$ and $\mathrm{HCO}_{3}^{-}$. Bone, 1999, 25(2): 107S-111S

30. Gondolph-Zink B. Effect of hydroxyapatite layering on the osteointegration of weightbearing and non-weightbearing implants. Comparison to other microporous surfaces in animal experiments. Orthopade, 1998, 27(2): 96-104

31. Layrolle P J F. Method for coating medical implants. US Patent, 6207218, 2001

32. Li P, Ducheyne P. Quasi-biological apatite film induced by titanium in a simulated body fluid. Journal of Biomedical Materials Research, 1998, 41(3): 341-348

33. Ono I, Gunji H, Kaneko F, et al. Efficacy of hydroxyapatite ceramic as a carrier for recombinant human bone morphogenetic protein. Journal of Craniofacial Surgery, 1995, 6(3): 238-244

34. Fiorellini J P, Buser D, Riley E, et al. Effect on bone healing of bone morphogenetic protein placed in combination with endosseous implants: a pilot study in beagle dogs. International Journal of Periodontics \& Restorative Dentistry, 2001, 21(1): 41-47

35. Kawai T, Mieki A, Ohno Y, et al. Osteoinductive activity of composites of bone morphogenetic protein and pure titanium. Clinical Orthopaedics and Related Research, 1993, 290: 296-305

36. Reddi A H, Cunningham N S. Bone induction by osteogenin and bone morphogenetic proteins. Biomaterials, 1990, 11: 33-34

37. Agrawal C M, Best J, Heckman J D, et al. Protein release kinetics of a biodegradable implant for fracture non-unions. Biomaterials, 1995, 16(16): 1255-1260

38. Ono I, Gunji H, Suda K, et al. Bone induction of hydroxyapatite combined with bone morphogenetic protein and covered with periosteum. Plastics and Reconstructive Surgery, 1995, 95(7): $1265-1272$

39. Esenwein S A, Esenwein S, Herr G, et al. Osteogenetic activity of BMP-3-coated titanium specimens of different surface texture at the orthotopic implant bed of giant rabbits. Chirurg, 2001, 72(11): 1360-1368

40. Wang X, Jin Y, Liu B L, et al. Tissue reactions to titanium implants containing bovine bone morphogenetic protein: a scanning electron microscopic investigation. International Journal of Oral and Maxillofacial Surgery, 1994, 23(2): 115-119

41. Endo K. Chemical modification of metallic implant surfaces with biofunctional proteins (Part 1). Molecular structure and biological activity of a modified NiTi alloy surface. Dental Materials Journal, 1995, 14(2): 185-198

42. Kim H M, Miyaji F, Kokubo T, et al. Preparation of bioactive Ti and its alloys via simple chemical surface treatment. Journal of Biomedical Materials Research, 1996, 32(3): 409-417

43. Urist M R. Bone formation by autoinduction. Science, 1965, 150: 893

44. Aldinger G, Herr G, Kusswetter W, et al. Bone morphogenetic protein: a review. International Orthopaedics, 1991, 15(2): 169-177

45. Elima K. Osteoinductive proteins. Annual Medicine, 1993, 25(4): 395-402

46. Lee M B. Bone morphogenetic proteins: background and implications for oral reconstruction. A review. Journal of Clinical Periodontology, 1997, 24(6): 355-365

47. Takahashi K. Bone morphogenetic protein (BMP): from basic studies to clinical approaches. Nippon Yakurigaku Zasshi, 2000, 116 (4): 232-240

48. Franceschi R T. The developmental control of osteoblast-specific gene expression: role of specific transcription factors and the extracellular matrix environment. Critical Reviews in Oral Biology \& Medicine, 1999, 10(1): 40-57

49. Yamaguchi A. Recent advances in research on bone formation-BMP action and its mechanism. Nippon Rinsho, 2002, 60S3: 40-47

50. Reddi A H. Initiation of fracture repair by bone morphogenetic proteins. Clinical Orthopaedics and Related Research, 1998, 355: S66-S72

51. Lee D D, Tofighi A, Aiolova M, et al. alpha-BSM: a biomimetic bone substitute and drug delivery vehicle. Clinical Orthopaedics and 
Related Research, 1999, 367: S396-S405

52. Schmidmaier G, Wildemann B, Cromme F, et al. Bone morphogenetic protein-2 coating of titanium implants increases biomechanical strength and accelerates bone remodeling in fracture treatment: a biomechanical and histological study in rats. Bone, 2002, 30(6): 816-822

53. Salata L A, Franke-Stenport V, Rasmusson L. Recent outcomes and perspectives of the application of bone morphogenetic proteins in implant dentistry. Clinical Implant Dentistry and Related Research, 2002, 4(1): 27-32

54. Hollinger J O, Leong K. Poly(alpha-hydroxy acids): carriers for bone morphogenetic proteins. Biomaterials, 1996, 17(2): 187-194

55. King G N. The importance of drug delivery to optimize the effects of bone morphogenetic proteins during periodontal regeneration. Current Pharmaceutical Biotechnology, 2001, 2(2): 131-142

56. Kirker-Head C A. Potential applications and delivery strategies for bone morphogenetic proteins. Advanced Drug Delivery Reviews, 2000, 43(1): 65-92

57. Liu Y, Hunziker E B, van de Vaal C, et al. Biomimetic coatings vs. collagen sponges as a carrier for BMP-2: a comparison of the osteogenic responses triggered in vivo using an ectopic rat model. Bioceramics 16, 2004, 254-256: 619-622

58. Liu Y, Hunziker E B, Layrolle P, et al. Bone morphogenetic protein 2 incorporated into biomimetic coatings retains its biological activity. Tissue Engineering, 2004, 10(1-2): 101-108

59. Liu Y. Introduction of ectopic bone formation by BMP-2 incorporated biomimetically into calcium phosphate coatings of titanium-alloy implants. Bioceramics 15, 2002, 240-242: 667-670

60. Oakes D A, Lieberman J R. Osteoinductive applications of regional gene therapy: ex vivo gene transfer. Clinical Orthopaedics and Related Research, 2000, 379: S101-S112

61. Ohgushi H, Caplan A I. Stem cell technology and bioceramics: from cell to gene engineering. Journal of Biomedical Materials Research, 1999, 48(6): 913-927

62. Scaduto A A, Lieberman J R. Gene therapy for osteoinduction. Orthopedic Clinics of North America, 1999, 30(4): 625-633

63. Alden T D, Varady P, Kallmes D F, et al. Bone morphogenetic protein gene therapy. Spine, 2002, 27(16): S87-S93

64. Becker W, Becker B E. Periodontal regeneration updated. Journal of the American Dental Association, 1993, 124(7): 37-43

65. Shirkhanzadeh M, Liu G Q. Biocompatible delivery systems for osteoinductive proteins: immobilization of L-lysine in microporous hydroxyapatite coatings. Materials Letters, 1994, 21: 115-118

66. Coombes A G, Heckman J D. Gel casting of resorbable polymers. 1. Processing and applications. Biomaterials, 1992, 13: 217-224

67. Liu Y, de Groot K, Hunziker E B. BMP-2 liberated from biomimetic implant coatings induces and sustains direct ossification in an ectopic rat model. Bone, 2005, 36(5): 745-757

68. Liu Y, Enggist L, Kuffer A F, et al. The influence of BMP-2 and its mode of delivery on the osteoconductivity of implant surfaces during the early phase of osseointegration. Biomaterials, 2007, 28 (16): 2677-2686 